\title{
Start-up scenario studies in gyrotron oscillator using a novel linear and spectral code
}

\author{
J. Genoud*, T. M. Tran*, S. Alberti*, P. Kaminski*, F. Braunmueller*, J.-Ph. Hogge*, M. Q. Tran*, \\ W. C. Guss ${ }^{\dagger}$ and R. J. Temkin ${ }^{\dagger}$. \\ ${ }^{*}$ Swiss Plasma Center, Ecole Polytechnique Fédérale de Lausanne (EPFL), Station13, CH-1015, Lausanne, Switzerland. \\ ${ }^{\dagger}$ Plasma Science and Fusion Center, Massachusetts Institute of Technology, Cambridge, MA 02139 USA
}

\begin{abstract}
A linear and spectral model has recently been developed [1], describing the self-consistent wave-particle interaction in a gyrotron oscillator. The spectral approach, compared to commonly used time-evolution approaches, has the possibility to describe all of the stable and unstable modes, respectively, with negative and positive growth rates. Moreover, this approach is numerically efficient and thus appropriate for parameter scans or start-up scenario studies. The model has been successfully benchmarked against real experiments for gyrotron cavity interaction, in particular concerning start-up scenario studies. In order to study backward-wave instabilities in smooth-wall beam ducts, the numerical model has been recently extended to include a higher order finite element discretization. The model, its numerical implementation and simulation results for high power gyrotrons as well as first results for smooth-wall beam ducts will be presented.
\end{abstract}

\section{THEORETICAL MODEL}

The linear model used in these simulations is based on the self-consistent non-linear model TWANG [2]. As described in [1], the linearized set of equations is converted into a closed system of three complex PDE's using a moment approach and is subsequently solved as a general eigenvalue problem after a Fourier transform in time. This model permits to simulate real systems, considering the main inhomogeneities in the cavity wall, magnetic field profiles and associated inhomogeneities of the electron beam properties. Recently the code has been adapted to treat backward-wave instabilities potentially occurring in smooth-wall beam-duct, replacing the finite difference method used for the discretization by a finite element method of arbitrary order, and adding a radiation boundary condition at the entry of the interaction region.

One of the main advantages of the spectral approach, compared to time-evolution approach, is the possibility to describe not only the most unstable mode but also all of the stable and unstable modes. The computation resources required are also lower for a spectral code, in particular for starting current calculations. Indeed, the computation time in a spectral code is independent on the physical parameters, whereas in a time-evolution code it depends on the wave growth rate and therefore on the different beam parameters. Such a spectral code is thus appropriate for start-up scenarios studies and also for beam-duct studies where large parameter scans are required.

\section{RESULTS}

As a fist application of the spectral approach, the study of the startup for the $1.5 \mathrm{MW}, 110 \mathrm{GHz}$ MIT-gyrotron is presented in figure 1. The starting current for three transverse modes is calculated during the start-up phase of the gyrotron, using the experimental data from [3] for the cathode voltage and electron pitch angle (figure $1 \mathrm{a}$ )). The red line in figure $1 \mathrm{~b}$ ), representing the experimentally measured beam current, first crosses the starting current curve of the $\mathrm{TE}_{22,6}$ modes, indicating that it is the first mode to be excited during the start-up phase. However, it has also been observed that these results are strongly dependent on the experimental parameters and that a precise knowledge on these parameters is required in order to have accurate simulation results. Using the novel spectral model and including the uncertainty on some experimental parameters, the start-up predicted by the model is consistent with the experimental results.
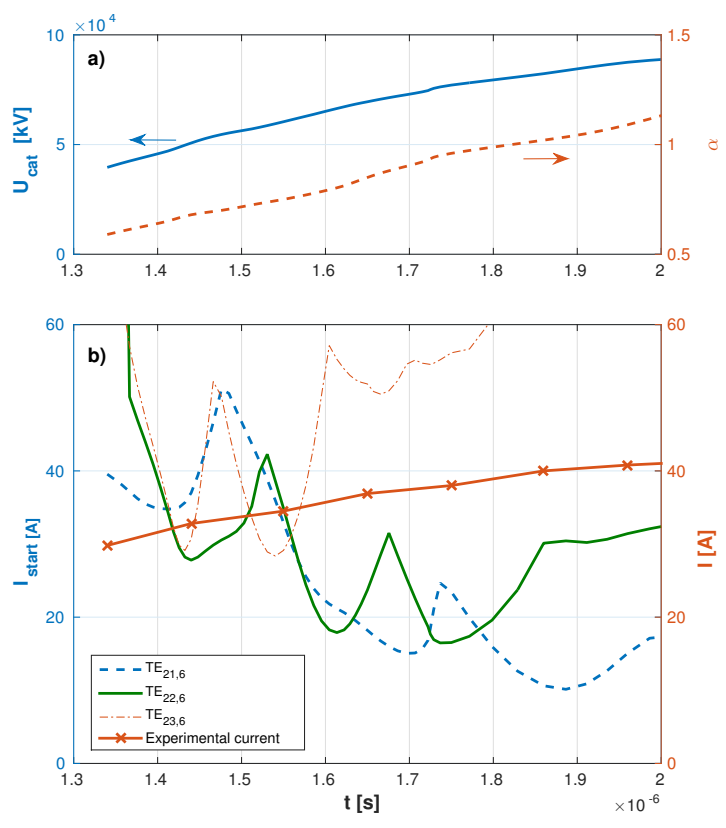

Fig. 1: a) Experimental traces of the cathode voltage (blue) and theoretical pitch angle (red, dashed) (from [3]). b) Starting current curves for three transverse modes calculated during the start-up phase. The cathode voltage and pitch angle values used for the computations are shown in a).

In addition to the cavity oscillation studies, the model can 
also be used to study parasitic backward-wave instabilities in smooth-wall gyrotron beam-ducts. For this particular case, the finite difference scheme used for the discretization showed some numerical limitations. Hence, the model has been extended with a hybrid finite element scheme of arbitrary order. As a preliminary study, the smooth-wall beam-duct described by Yu et al. [4] has been considered. The beam-duct geometry and the magnetic field profile used for the simulations are shown in figure 2. For the parameters listed in table I, the improvement in the complex eigenvalue convergence for a mode is shown in the frequency/growth-rate plane in figure 3 . While the advantage of using a higher order finite element discretization is clear in terms of convergence, it is found that the converged values are not the same for the two discretization. This discrepancy can be explained by the fact that the radiation boundary conditions which involve the derivative of the complex field at the two ends are best described with a finite element scheme. The corresponding eigenvector, described by the amplitude and phase of the rf-wave electric field are shown in figure 4 for the unstable mode calculated above. The electric field profile is mainly situated at the output part of the beam-duct, near the entry of the cavity. As expected, the phase profile corresponds to a backward wave.

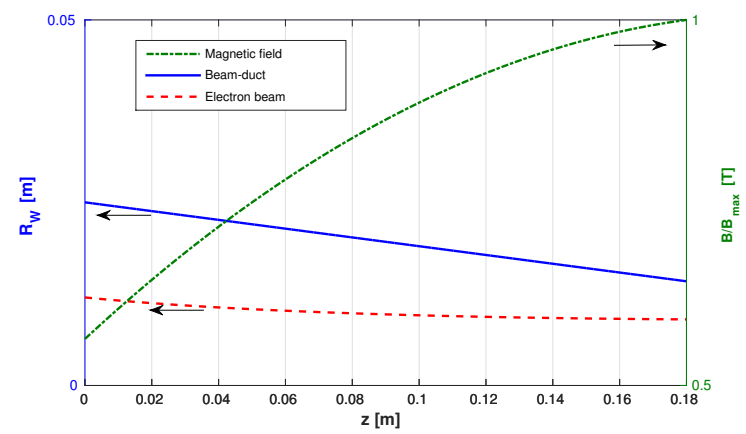

Fig. 2: Longitudinal profile of the beam-duct (in blue), position of the electron-beam (in red, dashed) and normalized magnetic field profile (in green, dashed-dotted)

\begin{tabular}{|l|c|}
\hline \multicolumn{1}{|c|}{ Parameter } & Value \\
\hline Transverse mode & $\mathrm{TE}_{22,7}$ \\
Beam radius & $9 \mathrm{~mm}$ \\
Pitch angle & 1.3 \\
Acceleration voltage & $80 \mathrm{kV}$ \\
\hline Magnetic field & $6.25 \mathrm{~T}$ \\
Beam current & $100 \mathrm{~A}$ \\
\hline
\end{tabular}

TABLE I: Simulation parameters. The magnetic field, beam radius and pitch angle values are set at the end of the interaction region.

In the future, the spectral code will be used both to continue start-up scenario studies, in particular for gyrotron where parasitic oscillations were observed during the start-up phase, and to study spurious instabilities in dielectric loaded smoothwall beam duct, starting from simple homogeneous beam-duct geometry and magnetic field profile and studying the effects of the wall or magnetic field tapering.

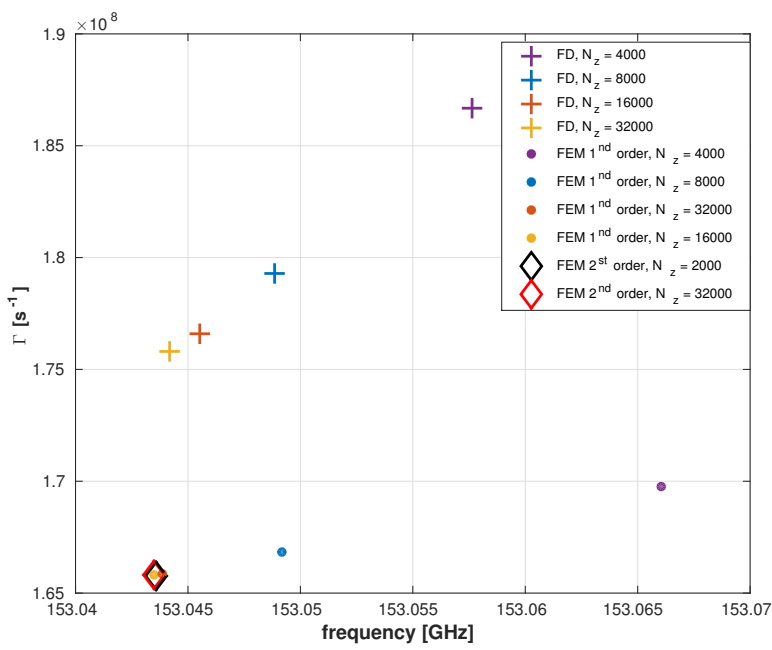

Fig. 3: Eigenvalue computed with the code TWANGlinspec for the two discretization methods and for different number of points $\mathrm{Nz}$ used for the discretization. The parameters are listed in table I.
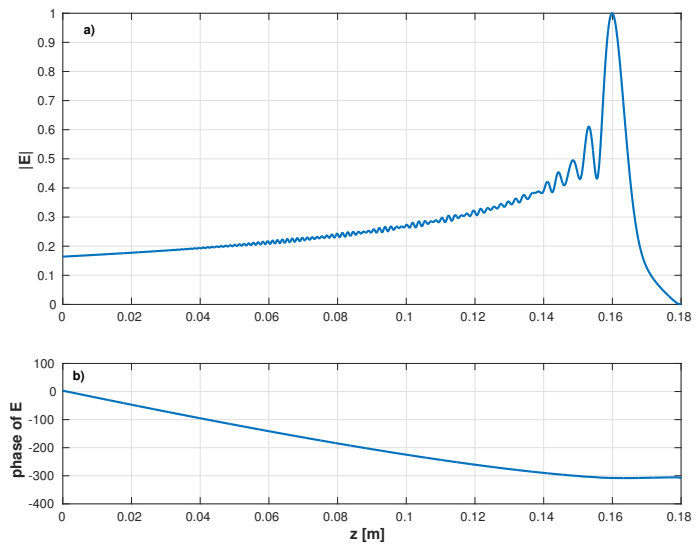

Fig. 4: Amplitude (a) and phase (b) of the electric field profile calculated with TWANGlinspec with a finite element method of second order and for the parameter listed in table I.

\section{ACKNOWLEDGMENTS}

This work has been partially carried out within the framework of the EUROfusion Consortium and has received funding from the Euratom research and training programme 2014-2018 under grant agreement No 633053, and within the framework of Fusion for Energy under Grant F4E-GRT-432 and -553 within the European Gyrotron Consortium (EGYC). The views and opinions expressed herein do not necessarily reflect those of the European Commission. EGYC is a collaboration of SPC, Switzerland; KIT, Germany; HELLAS, Greece and IFP-CNR, Italy. Work at MIT was supported by the US DOE Office of Fusion Energy Sciences under Grant DE-FC02-93ER54186.

\section{REFERENCES}

[1] J. Genoud et al., Physics of Plasmas, 23(4):043101, (2016).

[2] F. Braunmueller et al., Physics of Plasmas, 22(6):063115, (2015).

[3] D. S. Tax et al., IEEE Transactions on Plasma Science, 41(4):862-871 (2013).

[4] J. Yu et al., IEEE Transactions on Plasma Science, 38(6):1193-1199 (2010). 